\title{
An alternative approach to measure $\beta$-delayed neutron emission
}

\author{
Alexey Evdokimov*, Iris Dillmann, and Michele Marta \\ II. Physikalisches Institut, Justus-Liebig Universität Giessen and \\ GSI Helmholtzzentrum für Schwerionenforschung GmbH, Darmstadt, Germany \\ E-mail: a.evdokimovegsi.de, i.dillmann@gsi.de
}

Fritz Bosch, Alexey Dolinskii, Christophor Kozhuharov, Yuri Litvinov, Fritz Nolden, Markus Steck, and Helmut Weick

GSI Helmholtzzentrum für Schwerionenforschung GmbH, Darmstadt, Germany

\section{Thomas Faestermann, Roman Gernhäuser, and Ludwig Maier}

Physik Department E12, Technische Universität München, Germany

\begin{abstract}
A new method is proposed to determine half-lives and branching ratios of $\beta$-delayed neutron emitters, especially those beyond $N=126$ that are relevant for the $r$-process. The existing storage ring ESR or the future ring CR at GSI would be employed to store and cool the mother nuclei. The decay half-life and the neutron emission probability could be deduced from the detection of the mother and decay daughter ions with Schottky pick-ups and a particle detector in contrast to the standard method via detection of $\beta$-delayed neutrons. This method could be complementary and has the advantage to be independent of the neutron detection efficiency. As candidates for a proof-of-principle tests we suggest the nuclei ${ }^{211} \mathrm{Hg},{ }^{212} \mathrm{Hg},{ }^{210} \mathrm{Tl}$, and ${ }^{213} \mathrm{Tl}$ which have been investigated in a recent experiment by traditional detection methods.
\end{abstract}

XII International Symposium on Nuclei in the Cosmos,

August 5-12, 2012

Cairns, Australia

${ }^{*}$ Speaker. 


\section{Experimental status of neutron-rich isotopes}

The "rapid neutron capture" ( $r$ ) process plays an important role in stellar nucleosynthesis and is responsible for about $50 \%$ of the solar abundances beyond iron. The solar $r$-abundance curve has three maxima at $A \sim 80,130$, and 195, corresponding to neutron-rich precursors around $N=50$, 82, and 126 (Fig. 1). Up to now experiments in or close to the r-process path were carried out only for $A<150$, leaving the region above as (experimental) "terra incognita". Recent experiments at the FRS at GSI Darmstadt have led to the discovery of 105 new isotopes between $\mathrm{Nd}(Z=60)$ and $\mathrm{Ac}$ $(Z=89)[1-3]$. However, no physical properties like half-lives and masses have been measured yet for most of these new isotopes, and the $r$-process progenitors at the $N=126$ shell closure are still more than 10-15 mass units away from the presently last known isotopes. Further reduction of this gap and first measurements inside the $r$-process path at $A \approx 200$ is one of the prime motivation of all present and future radioactive-beam facilities.

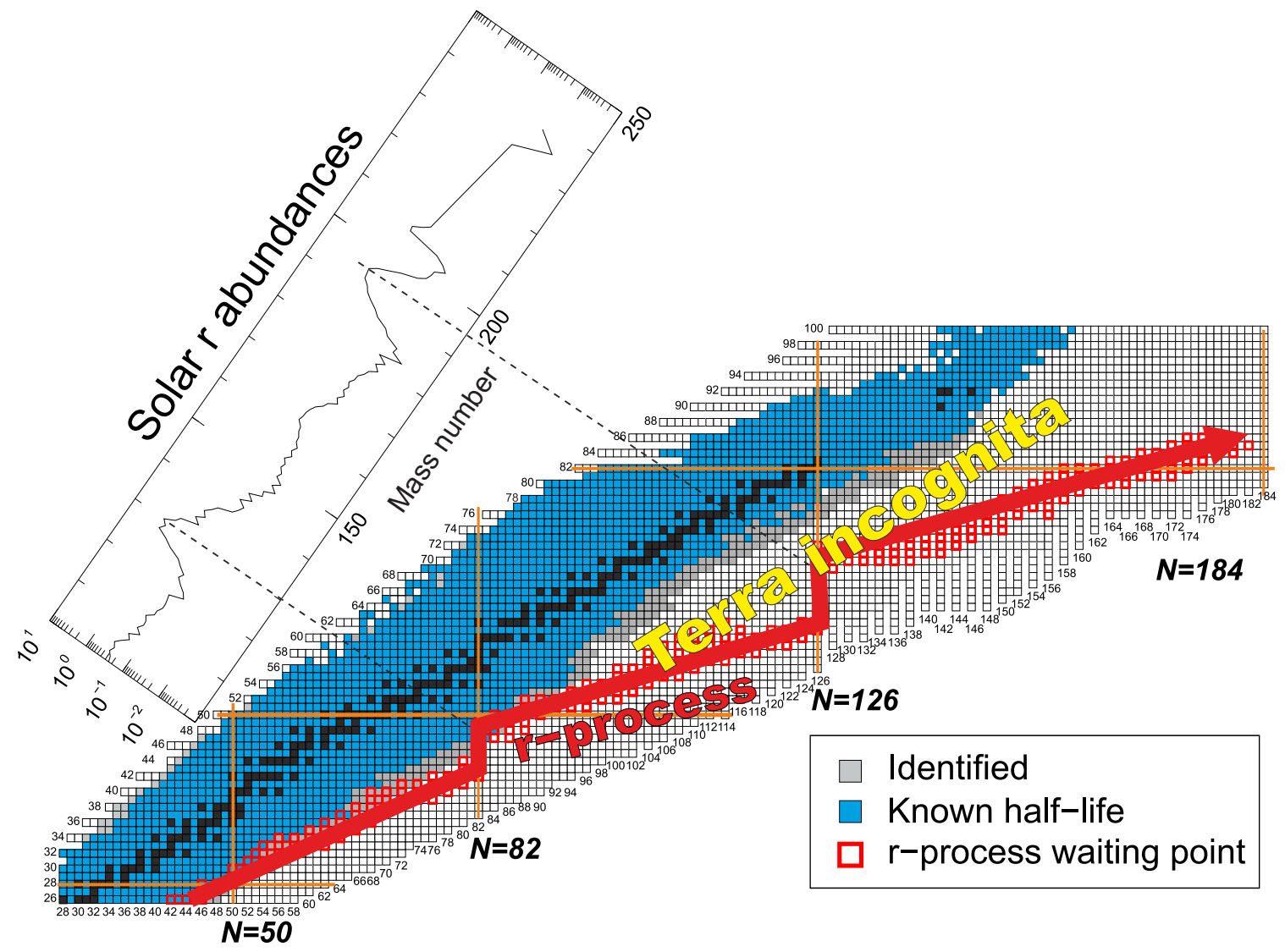

Figure 1: Chart of nuclides with $r$-process region.

$\beta$-delayed neutron emission occurs in very neutron-rich nuclei when the $\beta^{-}$-decay energy $\left(Q_{\beta}\right.$ value) is larger than the neutron separation energy $\left(S_{n}\right)$. The branching ratio $\left(P_{n}\right.$, neutron emission probability) is the number of emitted neutrons per $100 \beta$-decays. Measurements of $\beta$-delayed neutron $(\beta \mathrm{n})$ precursors have been carried out mainly for the mass region $A<150$, with the exception of ${ }^{210} \mathrm{Tl}\left(P_{n}=0.007_{-0.004}^{+0.007} \%\right)$ [4]. In an experimental campaign at the FRS 2011 the heaviest $\beta$ n-emitters so far in the mass region "south-east" of ${ }^{208} \mathrm{~Pb}$ have been measured [5]. Among these 


\begin{tabular}{|l||c|c|c|c|}
\hline & $\mathbf{t}_{1 / 2}$ (exp.) & $P_{n}$ (exp.) & $\mathbf{t}_{1 / 2}$ (theo.) [6, 7] & $P_{n}$ (theo.) [6, 7] \\
\hline \hline${ }^{211} \mathrm{Hg}$ & - & - & $2 \mathrm{~s} / 14.92 \mathrm{~s}$ & $7.35 \% / 0.8 \%$ \\
\hline${ }^{212} \mathrm{Hg}$ & - & - & $2.4 \mathrm{~s} / 10.5 \mathrm{~s}$ & $15.9 \% / 2.34 \%$ \\
\hline${ }^{210} \mathrm{Tl}$ & $1.30 \mathrm{~min}$ & $0.007 \%[4]$ & $-/ 57.53 \mathrm{~s}$ & $-/ 0.01 \%$ \\
\hline${ }^{213} \mathrm{Tl}$ & - & - & $70.4 \mathrm{~s} / 32.4 \mathrm{~s}$ & $2.93 \% / 13.3 \%$ \\
\hline
\end{tabular}

Table 1: Half-lives and $P_{n}$ value predictions [6,7] of selected isotopes for the ESR measurements. The predictions for ${ }^{210} \mathrm{Tl}$ from [6] are not available.

isotopes ${ }^{211} \mathrm{Hg},{ }^{212} \mathrm{Hg},{ }^{210} \mathrm{Tl}$, and ${ }^{213} \mathrm{Tl}$ are possible candidates for a proof-of-principle measurement in the storage ring ESR due to their expected long half-lives (Table 1).

\section{Alternative method to measure $\beta$ n-emission probabilities}

In this complementary approach the secondary beams from the FRS are injected into the ESR (or in the future at FAIR via the Super-FRS into the Collector Ring CR). This project is part of the ILIMA collaboration (Isomeric beams, LIfetimes and MAsses) [8], which aims to carry out measurements of nuclear masses and lifetimes of stored exotic nuclei at relativistic energies at the new SuperFRS-CR facility. The higher beam intensity will allow to investigate exotic short-lived nuclei like those in the "terra incognita" which are up to now not accessible. The ESR (CR) storage ring is able to accumulate, cool and detect the secondary separated beams from the FRS (SuperFRS). The cooling of the injected beam via stochastic and (only for the ESR) electron cooling is needed to decrease its transverse emittance. A short overview of the characteristic features of the ESR and the future CR are shown in Table 2. In Fig. 2 we show the lattice structure of the ESR and a sketch of the future FAIR project.

\begin{tabular}{|l||c|c|}
\hline Parameters & ESR [9] & CR [10, 11] \\
\hline \hline Circumference & $108.36 \mathrm{~m}$ & $221 \mathrm{~m}$ \\
\hline Max. B $\rho$ magnetic rigidity & $10 \mathrm{Tm}$ & $13 \mathrm{Tm}$ \\
\hline Max. kinetic energy of injected isotopes & $400 \mathrm{MeV} / \mathrm{u}$ & $740 \mathrm{MeV} / \mathrm{u}$ \\
\hline Revolution time per turn & $\approx 500 \mathrm{~ns}$ & $\approx 855 \mathrm{~ns}$ \\
\hline Horizontal acceptance & $300 \mathrm{~mm} \mathrm{mrad}$ & $200 \mathrm{~mm} \mathrm{mrad}$ \\
\hline Vertical acceptance & $100 \mathrm{~mm} \mathrm{mrad}$ & $200 \mathrm{~mm} \mathrm{mrad}$ \\
\hline Momentum acceptance & $\pm 1.5 \%$ & $\pm 1.5 \%$ \\
\hline Stochastic cooling time & $\approx 1-1.5 \mathrm{~s}$ & $\leq 1 \mathrm{~s}[12]$ \\
\hline Electron and stochastic cooling time & $\approx 6-7 \mathrm{~s}$ & $\leq 1 \mathrm{~s} \mathrm{(only} \mathrm{stochastic)}$ \\
\hline
\end{tabular}

Table 2: Main features of the ESR and future CR. In the FAIR project no electron cooling is foreseen for the CR, only for the HESR and later the NESR.

The standard (non-destructive) technique to study the masses and half-lives of stored (and cooled) ions is by time-resolved Schottky mass spectrometry [13-15]. Capacitive pick-up plates provide the revolution frequencies of the ions and allow their identification. When the ions decay, they change their mass and possibly their charge, and thus their revolution frequencies. However, 
with Schottky spectrometry it is only possible to study the daughter nuclei that remain within the acceptance of the rings. This restricts the method to mother-daughter pairs within an $A / q$ change of $\pm 1.5 \%$, but it can be complemented by particle detectors in pockets behind the dipole magnets to detect also $\beta^{-} / \beta \mathrm{n}$-daughter nuclei can hit detector so many timesoutside the acceptance. The positions for such particle detectors in the ESR and the future CR are indicated with arrows in Fig. 2.

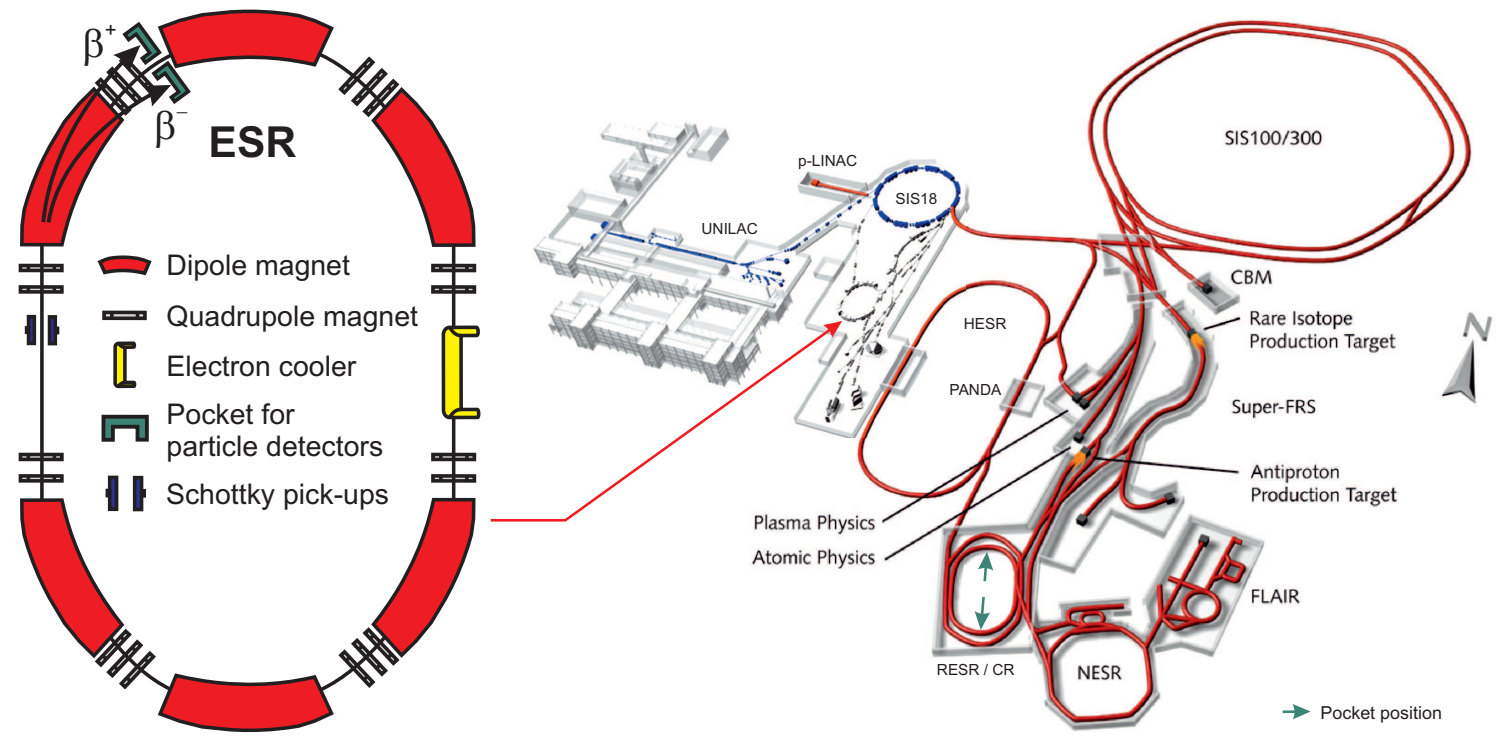

Figure 2: (Left) Lattice structure of the existing ESR. (Right) Sketch of FAIR with the planned positions for particle detectors in the CR (green arrows). The location of the ESR is marked by a red arrow.

The particle detector consists of a stack of silicon PIN-diodes and single- or double-sided silicon strip detectors (Fig. 3) which are mounted inside a stainless steel pocket with a 25-100 $\mu \mathrm{m}$ thick steel window [16]. This kind of detector telescope has already been used for the detection of ${ }^{207} \mathrm{~Pb}^{82+}$ ions in the bound-state $\beta$-decay experiment of ${ }^{207} \mathrm{Tl}^{81+}[17,18]$. $Z$ identification is accomplished by determination of the energy losses, and $\mathrm{X} / \mathrm{Y}$ position tracking is provided by the position-sensitive strip detectors. In order to measure decays outside of the acceptance, this detector is placed behind the dipole magnets in the arcs.

The use of such a pocket detector for the measurement of half-lives and $\beta$-delayed neutron branching ratios would have the advantage to be independent of the neutron energy and the neutron detection efficiency. This is - for the standard method with neutron detectors - the most critical part. Another advantage is that this measuring principle would be also independent of the very much restricted availability of ${ }^{3} \mathrm{He}$.

The detection efficiency of the particle detector in this setup is close to $100 \%$, but includes only those isotopes which decay on the long straight sections in the storage rings, whereas decays in the arcs result in losses of the ions. However, the overall efficiency is much lower due to unavoidable losses. Lower rates are expected compared to the direct measurements at the FRS (or Super-FRS) due to losses by the transfer into the ESR. Inside the storage rings collisions or electron pick-up processes have to be considered. Collisions of residual gas atoms with the circulating ion beam are negligible due to the ultra-high vacuum ( $\left.p \leq 1 \times 10^{-11} \mathrm{mbar}\right)$ operation. The main loss factor is 
radiative electron capture (REC) in the electron cooler. REC is a recombination process in which a highly ionized ion captures a quasifree electron and emits a photon. The REC processes of fully stripped ions can change substantially the storage lifetime of ions and subsequently the $P_{\beta}$ - and $P_{n}$-values. The loss rate by the REC processes depends on the electron density in the electron cooler and the revolution frequency of the ion beam. According to Refs. $[19,20]$ we calculated the loss rates for beams of the selected isotopes (see Table 1). These values are in the order of $\sim 10^{-3}-10^{-5} \mathrm{~s}^{-1}$ per ion for typical electron densities $\left(\sim 10^{5}-10^{7} \mathrm{~cm}^{-3}\right)$. REC processes lead also to a decrease of the stored beam lifetime and beam purity, which can give rise to background in the detector spectrum. For experiments using the electron cooler, the half-lives of the isotopes should be in the order of a few seconds (see Table 1 and 2). In the present ESR we plan to use only one pocket position (for $\beta \mathrm{n}$-measurements the inside position in Fig. 2), whereas in the future CR two positions are foreseen which would immediately double the total efficiency of this method. The monitoring of the number of circulating mother ions versus time with Schottky pick-ups and the simultaneous detection of the $\left(\beta^{-}\right.$and/or $\left.\beta \mathrm{n}\right)$ decay products with either the same pick-ups or the particle detector allows then to measure the half-lives and the $\beta$-delayed neutron branching ratio $\left(P_{n}\right.$-value).

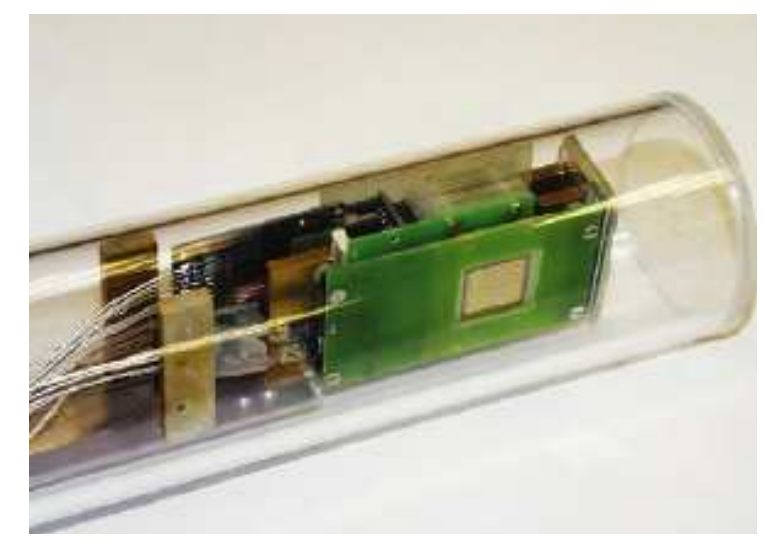

Figure 3: Previous particle detector used for storage ring experiments [18]. The active area was 40x60 $\mathrm{mm}^{2}$.

\section{Tracking simulations}

We have performed decay simulations for the ESR and CR rings with the codes MOCADI [21] and DYNAMO [22] in order to investigate the tracking of the decaying isotopes in the horizontal plane of the rings. The tracking simulations were done in $1^{\text {st }}$ order as "ideal" approach where only the ion optics and the aperture of magnets in the rings were considered. The particle detector was located as indicated by arrows in Fig. 2. $10^{4}$ ions of ${ }^{211} \mathrm{Hg}$ were injected and performed $10^{4}$ revolutions in the rings. The emittance of the injected beam was $1 \mathrm{~mm}$ mrad for the ESR and $5 \mathrm{~mm} \mathrm{mrad}$ for the CR which corresponds to the emittance of pre-cooled beams after stochastic cooling $[12,23]$. The mother ions $\left({ }^{211} \mathrm{Hg}\right)$ were always located in the center of the beamline at $0 \mathrm{~cm}$. The probabilities of $\beta^{-}-\left(P_{\beta}=92.65 \%\right)$ and $\beta$ n-decay $\left(P_{n}=7.35 \%\right)$ [6] were manually generated in the code. 

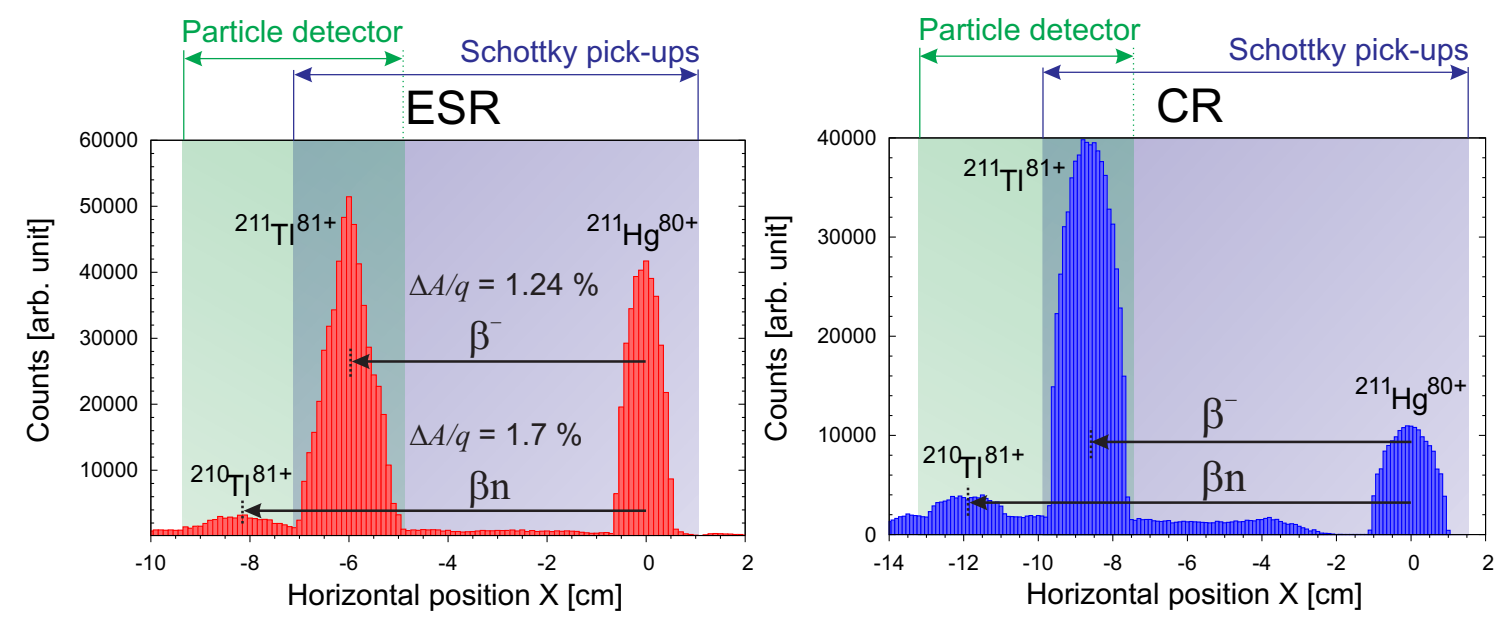

Figure 4: Tracking of ${ }^{211} \mathrm{Hg}$ and its decay products in the ESR (left) and CR (right) at the respective pocket positions. In accordance to Table 2 , the $\beta^{-}$-decay of ${ }^{211} \mathrm{Hg}$ to ${ }^{211} \mathrm{Tl}$ implies "small" change in $A / q$ of $1.24 \%$, which is still inside the acceptance of the ESR and CR. The $\beta$ n-decay to ${ }^{210} \mathrm{Tl}$ changes $A / q$ by $1.7 \%$, which would be outside the acceptance. The acceptance region for decay measurements by the particle detector and Schottky spectrometry are marked by green and violet backgrounds, correspondingly. With a $P_{n}$ of $7.35 \%$ and after $5 \mathrm{~ms} / 8.55 \mathrm{~ms}\left(10^{4}\right.$ revolutions in the ESR / CR $)$ all three components could be separated as shown.

The decays occur somewhere in the ring during the $10^{4}$ revolutions (corresponding to a duration of $5 \mathrm{~ms}$ and $8.55 \mathrm{~ms}$ for the ESR and CR, respectively). In the present stage of the simulations the influence of the different decay half-lives are not yet included. In Fig. 4 we show two examples for the simulation of the tracking of the mother ions $\left({ }^{211} \mathrm{Hg}\right)$ and the decay products $\left({ }^{211} \mathrm{Tl}\right.$ and ${ }^{210} \mathrm{Tl}$ ) in the ESR and CR. The separation between the orbits of the daughter and the mother ions are satisfactory for the chosen pocket positions. The detection efficiency is relatively high since almost $100 \%$ of the deflected ions (which are not lost otherwise) are detected by the particle detector. The relative numbers of surviving daughter and granddaughter ions after $10^{4}$ turns are $\sim 52 \%$ and $\sim 43 \%$ for the ESR and CR, respectively.

\section{Outlook}

We have presented a new method to measure $\beta \mathrm{n}$-branching ratios with storage rings. Proofof-principle tests at the existing ESR with ${ }^{211} \mathrm{Hg},{ }^{212} \mathrm{Hg}$, and ${ }^{213} \mathrm{Tl}$ are planned since they can be directly compared with recently measured values from the FRS [5]. Later on, this method will be extended to investigate well-known $\beta \mathrm{n}$-standards (e.g. ${ }^{87,88} \mathrm{Br},{ }^{94} \mathrm{Rb}$, and ${ }^{137} \mathrm{I}$ ). The planned particle detectors will be designed as multi-purpose detectors with a larger active area (up to 120x44 mm²) which can be used not only for $\beta$ n-measurements, but also for other decay channels like $\alpha$-, $n$ - and -decays.

\section{Acknowledgements}

Special thanks to Dr. Otto Klepper for sharing his incredible experience with us. This project is supported by Helmholtz Association via the Helmholtz Young Investigators Group LISA (VHNG 627) and by the German BMBF (NuSTAR-BMBF-FSP 302). 


\section{References}

[1] J. Kurcewicz et al., Phys. Lett. B 717, 371 (2012).

[2] H. Alvarez-Pol et al., Phys. Rev. C 82, 041602 (R) (2010).

[3] L. Chen et al., Nucl. Phys. A 882, 71 (2012).

[4] G. Stetter, Nucl. Sci. Abstr. 16, Abstr. 10963, 1409 (1962).

[5] R. Caballero-Folch et al., " $\beta$-delayed neutron emission measurements around the third r-process peak”, Proceedings of the XII. International Symposium on Nuclei in the Cosmos (NIC-XII), Cairns, Australia (August 2012), POS (NIC XII) 109.

[6] I. Borzov, private communication (2011).

[7] P. Möller et al., Phys. Rev. C 67, 055802 (2003).

[8] The NUSTAR-ILIMA collaboration, http://www.fair-center.de/de/fuer-nutzer/experimente/nustar/experiments/ilima.html .

[9] B. Franzke, Nucl. Instrum. Methods B 24/25, 18 (1987).

[10] A. Dolinskii et al., Nucl. Instrum. Methods A 532, 483 (2004).

[11] A. Dolinskii et al., Proceedings of the $23^{\text {rd }}$ Particle Accelerator Conference (PAC-09), Vancouver, British Columbia, Canada (May 2009), TH6PFP078, 3886 (2009), http://www.JACoW.org .

[12] C. Dimopoulou et al., Proceedings of the Workshop on Beam Cooling and Related Topics (COOL-11), Alushta, Ukraine (September 2011), TUIOB02, 58 (2011), http://www.JACoW.org .

[13] J. Borer, P. Brabham, H. G. Hereward, K. Hübner, W. Schnell, L. Thorndahl, Proc. 9th Int. Conf. on High Energy Accelerators (Stanford, CA), p.54 (1974).

[14] F. Nolden et al., Nucl. Instr. Meth. A 659, 69 (2011).

[15] B. Schlitt, PhD thesis, Ruprecht-Karls-Universität Heidelberg (1997).

[16] O. Klepper and C. Kozhuharov, Nucl. Instrum. Methods B 204, 553 (2003).

[17] T. Ohtsubo et al., Phys. Rev. Lett. 95, 052501 (2005).

[18] L. Maier, PhD thesis, Technische Universität München (2007).

[19] M. Steck et al., Proceedings of the $3^{\text {rd }}$ European Particle Accelerator Conference (EPAC-92), Berlin, Germany (March 1992), 827 (1992), http://www.JACoW.org .

[20] H. Poth, Phys. Rep. 196, 135 (1990).

[21] N. Iwasa et al., Nucl. Instr. Meth. B 126, 284 (1997).

[22] A. Dolinskii, private communications (2012).

[23] F. Nolden et al., Nucl. Phys. A 626, 491 (1997). 